\title{
Shape-shifting serpins
}

\section{Recent structures of members of the serine proteinase inhibitor families (serpins) continue to emphasize the flexibility of the reactive loop and suggest possible 'active' conformations.}

Proteinases are essential for the life cycles of all organisms, carrying out vital functions such as precursor processing, digestion, defence and degradation. But if such molecules were given a free rein they would perpetrate all kinds of unwanted damage and destruction. So it is not surprising that for most proteinases there is a proteinase inhibitor that controls the activity of these vital, and potentially deadly, enzymes.

The ubiquitous serine-proteinase inhibitors (serpins for short) constitute a family of such inhibitors : one of the most abundant proteins in beer - claimed to be responsible, in part, for its taste - is a barley serpin and one in ten proteins in our blood plasma is a serpin. Serpins regulate proteinases involved in a range of key biological processes that include blood coagulation, fertilization and viral inhibition of inflammation; indeed it is not surprising that serpin dysfunction is associated with lung, liver and blood coagulation diseases such as emphysema, liver cirrhosis, thrombosis and pulmonary embolism. Their mode of action is therefore of more than academic interest. Now two new serpin structures, a modified human antichymotrypsin reported in this month's Nature Structural Biology $y^{1,2}$, and a non-covalent dimer of antithrombin III molecules ${ }^{3,4}$, have moved us closer to a picture of the active conformation of serpins and an understanding of the features of the inhibition reaction.

Like other protein inhibitors of the serine proteinases ${ }^{5}$, the business end of a serpin is a short 15-residue peptide - the reactive loop - on the surface of the molecule and is principally responsible for interacting with the active site of the target serine proteinase. The serpin forms a stable covalent complex with the proteinase which can then be removed from the extracellular milieu by binding to specific cellular receptors. Alternatively, the cleaved and inactivated serpin may be slowly released from the complex.

Also in this month's Nature Structural Biology: critical residues for $\mathrm{SH} 3$ domain/peptide interactions; new ligandbinding pathways in myoglobin; haem doming as the primary event in haemoglobin ligand binding; structural dynamics in an electron-transfer complex; calbindin $\mathrm{D}_{9 \mathrm{~K}}$ in the absence of $\mathrm{Ca}^{2+}$; dendrotoxin-I structure; $a b$ initio prediction of a lysozyme-antibody complex; and are there knots in proteins?
The structures of inhibitory serpins reveal a radical transformation upon cleavage ${ }^{6,7}$. The reactive loop on the surface is cut between residues $\mathrm{P} 1$ (on the $\mathrm{N}$ terminal side of the loop) and $\mathrm{P} 1^{\circ}$ (on the $\mathrm{C}$-terminal side of the cleaved loop). The P1 side of the cleaved reactive loop then inserts into a large five-stranded $\beta$ sheet-sheet A-to become strand s4A, forming a six-stranded sheet, while the residues on the $\mathrm{P} 1^{\circ}$ side contribute to the smaller $\beta$-sheet $C$. Once contiguous amino acids in the intact molecule, the P1 and $\mathrm{P} 1^{\circ}$ residues are now up to $70 \AA$ apart in the cleaved molecule.

Not all serpins are inhibitory and the structure of one such non-inhibitory serpin, ovalbumin, has provided a first glimpse of the intact peptide which is the analogue of the reactive loop of the inhibitory serpins ${ }^{6,7}$. In this protein the loop forms a regular $\alpha$-helix, held above the surface of the protein on two short 'stalks'. In this conformation it is not a suitable substrate for the active site of a serine proteinase. Why can't the ovalbumin loop take on an extended conformation that would bind serine proteinases? Isolated peptides of varying length bind to and insert in the A-sheet in the normal position of the $\mathrm{P} 1-16$ reactive loop segment in inhibitory serpins, indicating that partial or pre-insertion of residues up to $\mathrm{P} 14$ in the $s 4 \mathrm{~A}$ strand is required for inhibitor function. A series of bulky residues in the $\mathrm{N}$-terminal stalk of ovalbumin reduces the flexibility of this region and prevents reinsertion of the surface peptide back into sheet $A$.

The conformational versatility of this family of molecules is further demonstrated by the structure of the 'latent', inactive form of human plasminogen-activator inhibitor-1. Here the reactive loop is fully inserted into the A-sheet as strand s4A even though the molecule has not been cleaved ${ }^{6,7}$. But until now what has remained tantalizingly elusive is the structure of the so-called 'active' conformation, the form that interacts with the target serine proteinases.

In the antithrombin dimer ${ }^{3,4}$ the reactive loop of one monomer is inserted into the $C \beta$-sheet of the other monomer, the latter having a cleaved/latent conformation. The reactive loop in the inserted monomer is in a conformation that is similar, but not identical, to that of other peptide-based proteinase inhibitors.

The $\mathrm{N}$-terminal stalk is partially inserted up to residue P14 in the A-sheet between strands $55 a$ and $s 3 a$.

How closely this mimics the real serpin-proteinase complex remains to be seen. Certainly it provides a framework for thinking about the antithrombinthrombin interaction. But some features are at odds with expectations, for example the $\mathrm{P} 1$ residue, arginine 393 , is pointing back towards the parent molecule rather than out towards the target molecule, and heparin, a potent stimulatory cofactor for the interaction that binds antithrombin, is not included in the complex.

The structure from Wei and colleagues ${ }^{1}$ provides a further twist in this tale of proteinase inhibitors. In this uncleaved and uncomplexed serpin, a variant of anti-chymotrypsin, a reactive loop is revealed with a very distorted helical structure. The active-site $\mathrm{P} 1$ residue, important in specificity and activity, is pointing out into solution, away from the serpin scaffold. As such, the primary recognition event could be docking with the proteinase $\mathrm{S} 1$ subsite.

Part of the N-terminal stalk, the equivalent of which is inserted in the active molecule in the antithrombin complex, is not pre-inserted, although there is an inviting $5 \AA$ gap at the 'top' of strands s5A and a3A. Wei et al. speculate that as the interaction proceeds, the reactive loop uncoils so that part of the stalk can then be inserted into the A-sheet, an idea consistent with immunological studies suggesting that insertion occurs after the serpinproteinase complex forms. At some point, the active-site covalent link locks the two molecules together. When the serpin is subsequently released it will adopt the inactive conformation.

There is still a way to go. Further details of the reaction coordinate need to be defined, as do the roles of the various cofactors in the interaction.

Guy Riddihough

Guy Riddihough is Editor of Nature Structural Biology

1. Wei, A., Rubin, H., Cooperman, B.S. \& Christianson D.W. Nature struct. Biol. 1, 250-257 (1994).

. Fletterick, R.J. \& McGrath, M.E. Nature struct. Biol. 1 201-203 (1994).

3. Schreuder, H.A. et al. Nature struct. Biol. 1, 48-54 (1994).

4. Carrell, R.W., Stein, P.E., Fermi, G. \& Wardell, M.R. Structure (in the press)

5. Bode, W. \& Huber, R. Curr. Opin. struct. Biol. 2, 45-52 (1991).

6. Carell, R.W. \& Evans, D.L.I. Curr. Opin. struct. Biol. 2 , 438-446 (1992).

7. Engh, R.A., Schulze, A.J., Huber, R. \& Bode, W. Behring Inst. Mitt. 93, 41-62 (1993). 